\title{
[F18] FDG-PET/CT for manual or semiautomated GTV delineation of the primary tumor for radiation therapy planning in patients with esophageal cancer: is it useful?
}

\author{
Franziska Walter ${ }^{1}$ (D) Constanze Jell ${ }^{1} \cdot$ Barbara Zollner $^{1} \cdot$ Claudia Andrae $^{1} \cdot$ Sabine Gerum $^{2} \cdot$ Harun Ilhan $^{3} \cdot$ \\ Claus Belka · Maximilian Niyazi · Falk Roeder ${ }^{2}$
}

Received: 8 June 2020 / Accepted: 29 September 2020 / Published online: 26 October 2020

(c) The Author(s) 2020

\begin{abstract}
Background Target volume definition of the primary tumor in esophageal cancer is usually based on computed tomography (CT) supported by endoscopy and/or endoscopic ultrasound and can be difficult given the low soft-tissue contrast of CT resulting in large interobserver variability. We evaluated the value of a dedicated planning [F18] FDG-Positron emission tomography/computer tomography (PET/CT) for harmonization of gross tumor volume (GTV) delineation and the feasibility of semiautomated structures for planning purposes in a large cohort.

Methods Patients receiving a dedicated planning [F18] FDG-PET/CT (06/2011-03/2016) were included. GTV was delineated on $\mathrm{CT}$ and on PET/CT $\left(\mathrm{GTV}_{\mathrm{CT}}\right.$ and $\mathrm{GTV}_{\mathrm{PET} / \mathrm{CT}}$, respectively) by three independent radiation oncologists. Interobserver variability was evaluated by comparison of mean GTV and mean tumor lengths, and via Sørensen-Dice coefficients (DSC) for spatial overlap. Semiautomated volumes were constructed based on PET/CT using fixed standardized uptake values (SUV) thresholds (SUV30, 35, and 40) or background- and metabolically corrected PERCIST-TLG and Schaefer algorithms, and compared to manually delineated volumes.

Results 45 cases were evaluated. Mean $\mathrm{GTV}_{\mathrm{CT}}$ and $\mathrm{GTV}_{\mathrm{PET} / \mathrm{CT}}$ were 59.2/58.0 ml, 65.4/64.1 ml, and 60.4/59.2 ml for observers A-C. No significant difference between CT- and PET/CT-based delineation was found comparing the mean volumes or lengths. Mean Dice coefficients on CT and PET/CT were 0.79/0.77, 0.81/0.78, and 0.8/0.78 for observer pairs $\mathrm{AB}, \mathrm{AC}$, and $\mathrm{BC}$, respectively, with no significant differences. Mean GTV volumes delineated semiautomatically with SUV30/SUV35/SUV40/Schaefer's and PERCIST-TLG threshold were 69.1/23.9/18.8/18.6 and 70.9 ml. The best concordance of a semiautomatically delineated structure with the manually delineated $\mathrm{GTV}_{\mathrm{CT}} / \mathrm{GTV}_{\mathrm{PET} / \mathrm{CT}}$ was observed for PERCIST-TLG.

Conclusion We were not able to show that the integration of PET/CT for GTV delineation of the primary tumor resulted in reduced interobserver variability. The PERCIST-TLG algorithm seemed most promising compared to other thresholds for further evaluation of semiautomated delineation of esophageal cancer.
\end{abstract}

Keywords Radiotherapy · PERCIST-TLG · Contouring · Gross tumor volume · Interobserver variability

Availability of data and materials The datasets used and/or analyzed during the current study are available from the corresponding author on reasonable request.

$\bowtie$ Franziska Walter

franziska.walter@med.uni-muenchen.de

1 Department of Radiation Oncology, University Hospital, Ludwig-Maximilians-University, Munich, Germany

2 Department of Radiotherapy and Radiation Oncology, Paracelsus Medical University (PMU), Landeskrankenhaus, Salzburg, Austria

3 Department of Nuclear Oncology, University Hospital, Ludwig-Maximilians-University, Munich, Germany

$\begin{array}{ll}\text { Abbreviations } \\ \text { CRT } & \text { Chemoradiotherapy } \\ \text { CT } & \text { Computed tomography } \\ \text { DSC } & \text { Sørensen-Dice coefficient } \\ \text { GEJ } & \text { Cancer of the esophagogastric junction } \\ \text { GTV } & \text { Gross tumor volume } \\ \text { GTV } & \text { Gross tumor volume derived by CT } \\ \text { GTV } & \text { GET/CT } \\ \text { IMRT } & \text { Intensity-modulated radiotherapy } \\ \text { LoD } & \text { Length of disease }\end{array}$


Fig. 1 Manual gross tumor volume (GTV) delineation by three observers (yellow, blue, green) a on CT alone axial and b sagittal; c GTV delineation on fused PET/CT axial and d sagittal
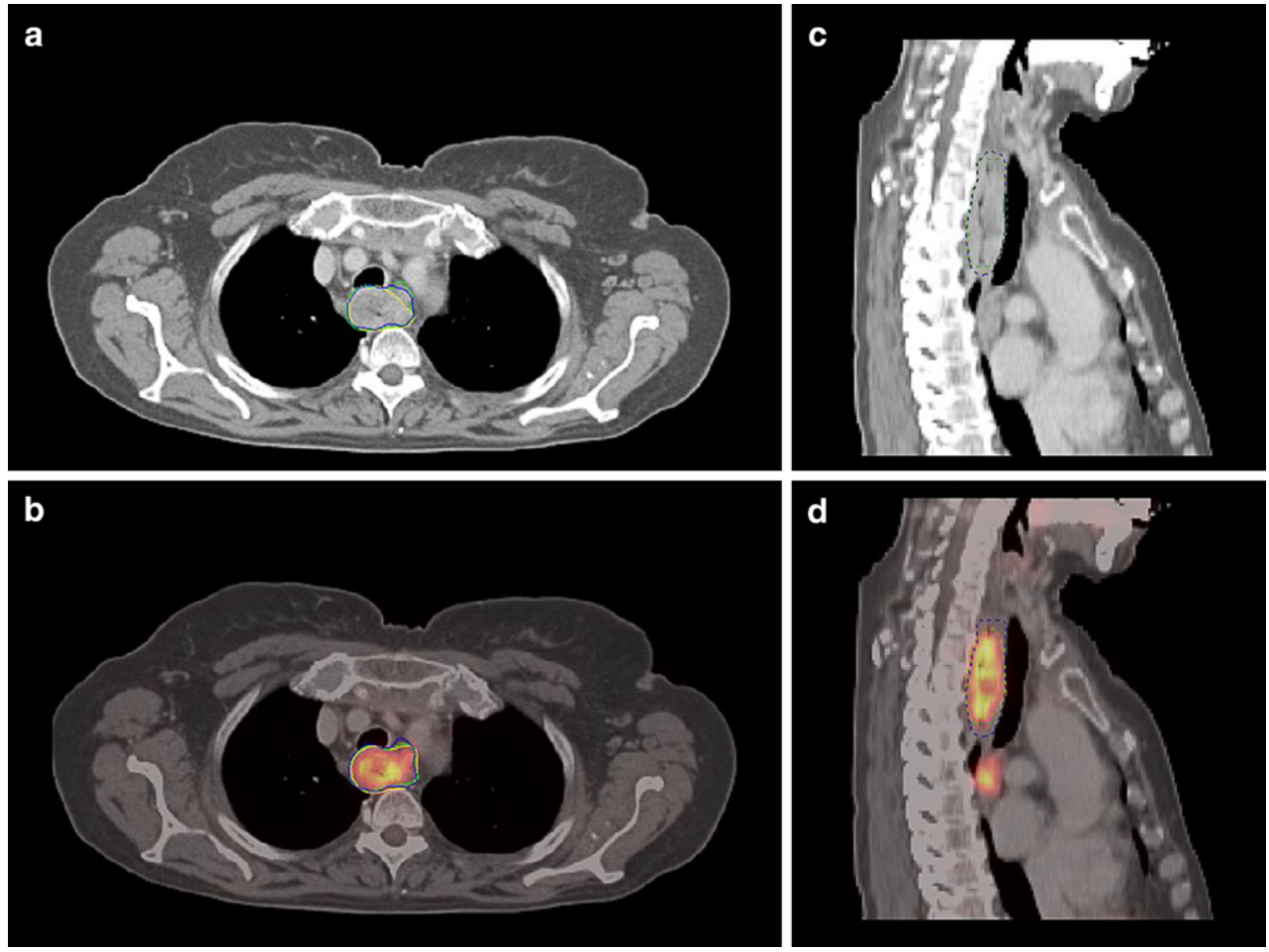

$\begin{array}{ll}\text { PET/CT } & \begin{array}{l}\text { Positron-emission tomography/computer } \\ \text { tomography }\end{array} \\ \text { PTV } & \begin{array}{l}\text { Planning target volume } \\ \text { SPSS }\end{array} \\ \text { Statistical Package for Social Sciences } \\ \text { SUV }_{\max } & \text { Standardized uptake values } \\ \text { VMAT } & \text { Maximum standardized uptake value } \\ & \text { Volumetric modulated arc therapy }\end{array}$

\section{Introduction}

Radiation therapy is a cornerstone of the multimodality treatment of locally advanced esophageal cancer [1, 2], either as definitive chemoradiotherapy (CRT) or as preoperative CRT based on randomized controlled trials [3, 4]. While dose and fractionation concepts have remained relatively constant, a considerable shift towards smaller target volumes resulting in less toxicities has taken place over the past decades. For example, the landmark trial RTOG 85-11, which established concurrent CRT as the standard of care for inoperable locally advanced esophageal cancer in the early 90s, had used generous margins for elective nodal irradiation as well as for a tumor bed boost [3]. In contrast, the CROSS trial establishing the role of neoadjuvant CRT more than a decade later introduced much smaller margins including the GTV without any elective nodal irradiation [4]. Similar (smaller) approaches have been proposed recently by the Expert Consensus Contouring guidelines by Wu et al. [5]. Therefore, accurate delineation of gross tumor volume becomes more crucial, especially when combined with the steep dose gradients achieved by modern radiation techniques like intensity-modulated radiation therapy.

CT-based target delineation still represents the standard of care for radiation therapy treatment planning of esophageal cancer patients, although precise definition of the exact tumor boundaries can be difficult, especially in the cranio-caudal direction but also towards directly adjacent mediastinal structures. PET/CT offers some general advantages compared to $\mathrm{CT}$ as it adds metabolic to anatomic information, thus enabling a more precise differentiation between vital tumor volume and surrounding structures. Therefore, it has been extensively used in diagnosis, staging, and response assessment of cancer patients [6-8]. For radiation therapy planning, PET/CT seems particularly interesting if performed in the treatment position and has been shown to impact target volumes, for example, in lung cancer, head and neck cancer, prostate cancer, and brain tumors [9-12]. While the role of PET/CT in assessment of lymph node involvement of esophageal cancer is well established [13, 14], its role in target delineation of the primary tumor is more controversial. Several studies have reported conflicting results [6, 15-20], which may have been affected by small sample sizes and different methods of PET/CT-based contouring. While most studies used visual interpretation of PET/CT images, others promoted either fixed absolute SUV thresholds, like SUV2.5 or percentages of SUVmax such as SUV20, with varying results $[15,16,21]$. Recently, our group reported even more 
Fig. 2 Manual gross tumor volume (GTV) delineation on a CT alone, $\mathbf{b}$ fused [F18] FDG-PET/CT, $\mathbf{c}$ GTV $_{\mathrm{CT}}$ (yellow), d GTV $\mathrm{PET}_{\text {CT }}$ (blue), e matched $\mathrm{GTV}_{\mathrm{CT}}$ (yellow), GTVPET/CT (blue), and PERCIST-TLG (red), f PERCIST-TLG (red) semiautomated contour
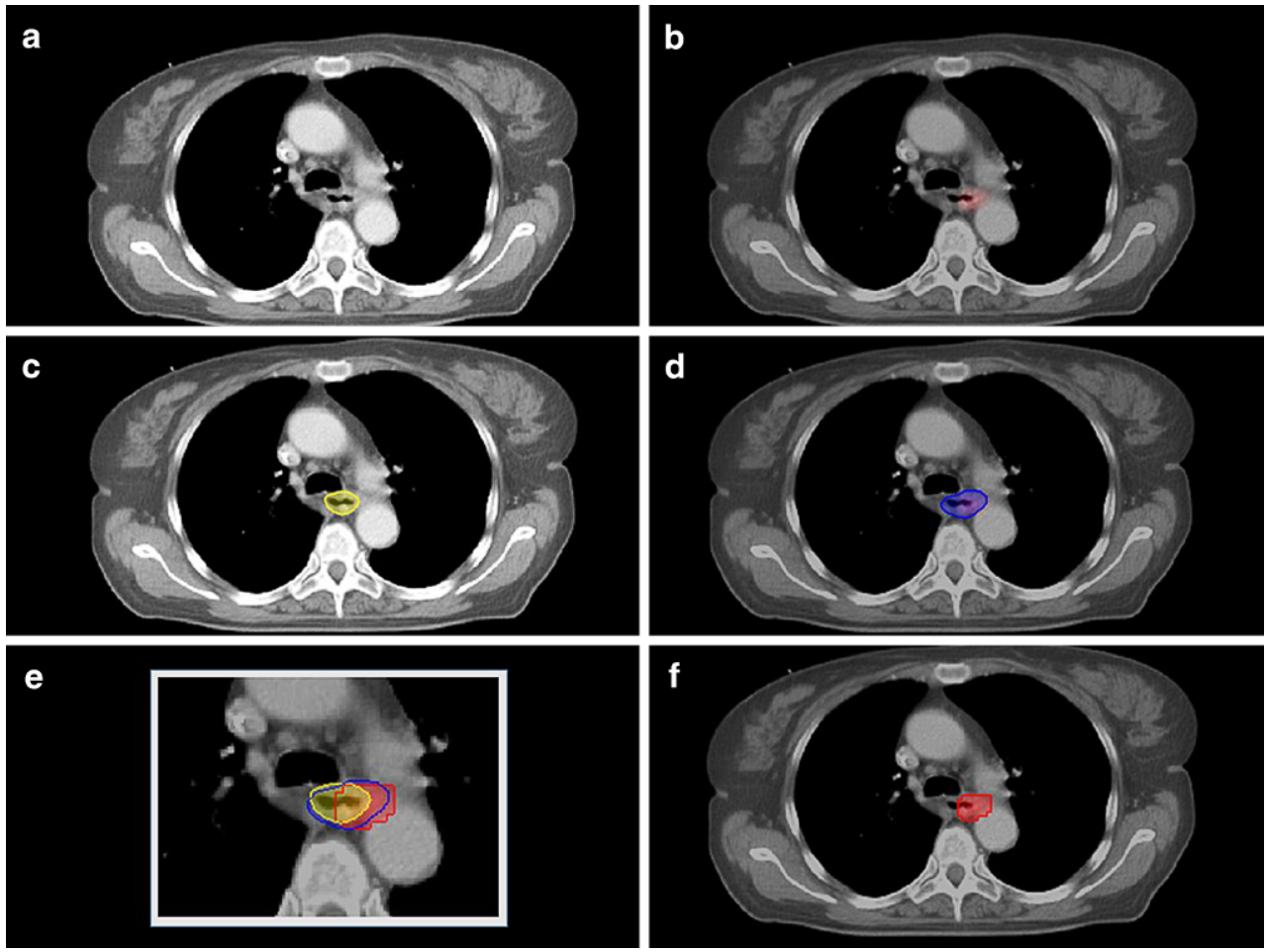

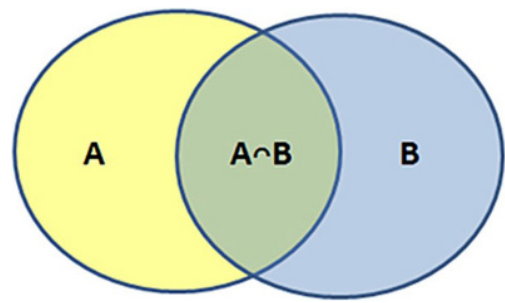

Fig. 3 Sørensen-Dice coefficient

complex background- and metabolically corrected values such as the PERCIST-TLG algorithm as most promising in a study including solid tumors of different origin [10].

Therefore, the purpose of the current study was to evaluate the value of additional PET/CT information on target delineation of gross tumor volume of the primary tumor in esophageal cancer patients in a larger patient cohort. The first part was designed to answer the question of whether the addition of visually interpreted PET/CT information to the standard CT-based planning approach can result in reduced interobserver variability for manual GTV definition taken as a surrogate for more accurate delineation. The second part should answer the question of whether semiautomatic delineation methods may help in the harmonization of GTV delineation, and which particular method should be preferred.

\section{Methods}

\section{Patients}

We retrospectively identified 57 consecutive patients who had been treated with neoadjuvant or definitive radiation therapy for non-metastatic esophageal cancer at our department with a dedicated PET/CT for treatment planning available from 6/2011 to 3/2016. Of these patients, 12 had to be excluded from the analysis, 6 for low [F18] FDG activity (maximum standardized uptake value, $\mathrm{SUV}_{\max },<4$ ), 2 scans showed artifacts from metallic material in the region of the tumor, and in 4 patients the software was unable to generate a semiautomated structure set. Therefore, 45 patients were included in the current study.

\section{[F18] FDG-PET/CT}

All included patients had received a dedicated planning [F18] FDG-PET/CT in supine treatment positioning prior to clinical treatment. Whole-body [F18] FDG-PET/CT scans were acquired from the base of the skull to the proximal femora (GE Discovery 690, General Electric, Munich, Germany). Patients fasted for at least $6 \mathrm{~h}$ before PET/CT images were acquired. Emission scans were initiated after a median uptake time of $60 \mathrm{~min}$ (range 46-113 min) following intravenous administration of $20 \mathrm{mg}$ of furosemide, $20 \mathrm{mg}$ of butylscopolamine, and [F18] FDG (mean activity $246 \mathrm{MBq}$, range: $184-322 \mathrm{MBq}$ ). Diagnostic CT images using intra- 
Fig. 4 a CT alone, b fused [F18] FDG-PET/CT, c SUV30 semiautomated contour (yellow), d PERCIST semiautomated contour (red), e matched SUV30 (yellow) and PERCIST (red)
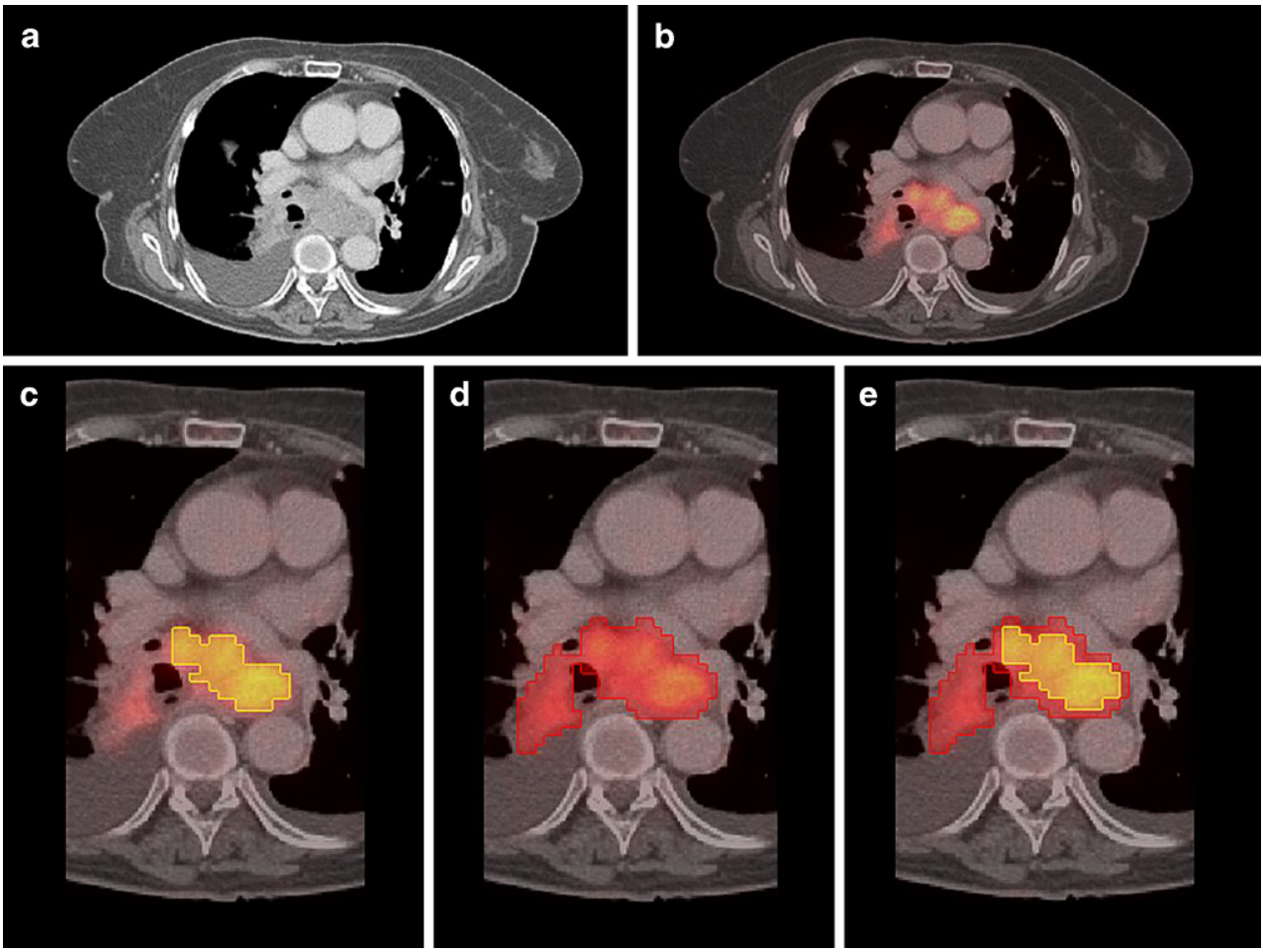

venous contrast agent in portal venous phase were acquired in suitable patients. PET images were reconstructed using ordered subset expectation maximization (OSEM)-based algorithms (VUE point FX).

\section{Manual gross tumor volume delineation}

Primary tumor delineation was performed by three independent experienced radiation oncologists without prior knowledge of the images or the clinically used target volumes both on PET/CT and CT only. Scans were blinded; only baseline clinical information including TNM stage and tumor extent derived by endoscopy was provided. The radiation oncologists were asked to define the GTV of the primary tumor and include affected lymph nodes only if directly adjacent to the main tumor, since these lymph nodes would also be included in the semiautomatically delineated GTVs. No delineation of nodal volumes (except the above mentioned) or clinical target volumes was performed. GTV of the primary tumor was defined for all studies by each radiation oncologist first on CT scans only $\left(\mathrm{GTV}_{\mathrm{CT}}\right)$. To avoid bias in contouring of the PET/CT-based GTV, definition of the respective GTVs on CT including the PET information $\left(\mathrm{GTV}_{\mathrm{PET} / \mathrm{CT}}\right)$ was done with delay and in random order (Fig. 1). For the manually delineated $\mathrm{GTV}_{\mathrm{PET} / \mathrm{CT}}$, no fixed SUV threshold was provided (visual interpretation; Fig. 2).

\section{Interobserver variability}

Mean tumor volumes and mean tumor lengths of the three observers were calculated per patient for each modality (CT and PET/CT) and compared in non-parametric paired analysis using the Wilcoxon test. To assess geometrical differences, Sørensen-Dice coefficients (Fig. 3) were computed for all three pair of observers and for each modality. Mean dice coefficients of the three observer pairs were calculated for each patient and each modality and compared by the Wilcoxon test.

\section{Semiautomated gross tumor volume delineation}

Semiautomated GTV delineation based on the given [F18] FDG-PET was performed using a dedicated software package (Hybrid Viewer, Hermes Medical Solutions, Stockholm, Sweden). Semiautomated GTVs were defined for a set of standardized uptake values (SUV) derived from the maximal SUV ( $\left.\mathrm{SUV}_{\max }\right)$ : SUV30, SUV35, and SUV40 defined as 30, 35, and $40 \%$ of SUV $_{\max }$. PERCISTTLG threshold was determined in analogy to the PERCIST criteria based on normal [F18] FDG background activity in a standardized $15 \mathrm{ml}$ VOI in the right hepatic lobe as described by Niyazi et al. [10]. Schaefer's threshold was calculated by using the formula $\mathrm{TS}=a \times \mathrm{SUV} 70+b \times \mathrm{BG}$ as described by Schaefer et al. ([22]; Fig. 4). 
Table 1 Patient characteristics

\begin{tabular}{|c|c|}
\hline Patient characteristics & \\
\hline \multicolumn{2}{|l|}{$\overline{\text { Gender }}$} \\
\hline Male & 32 \\
\hline Female & 13 \\
\hline \multicolumn{2}{|l|}{ Age } \\
\hline Median & 69 years \\
\hline Range & $53-85$ years \\
\hline \multicolumn{2}{|l|}{ Histology } \\
\hline Adenocarcinoma & 5 \\
\hline SCC & 39 \\
\hline Neuroendocrine carcinoma & 1 \\
\hline \multicolumn{2}{|l|}{ Localization } \\
\hline Cervical & 7 \\
\hline Upper thoracic & 16 \\
\hline Middle thoracic & 13 \\
\hline Lower thoracic/GEJ & 9 \\
\hline \multicolumn{2}{|l|}{ Grading } \\
\hline G1 & 3 \\
\hline $\mathrm{G} 2$ & 28 \\
\hline G3 & 14 \\
\hline \multicolumn{2}{|l|}{ Tstage } \\
\hline $\mathrm{cT} 2$ & 5 \\
\hline cT3 & 30 \\
\hline cT4 & 10 \\
\hline \multicolumn{2}{|l|}{ N stage } \\
\hline No & 16 \\
\hline $\mathrm{N}+$ & 29 \\
\hline \multicolumn{2}{|l|}{ RT technique } \\
\hline 3D-CRT & 29 \\
\hline IMRT & 16 \\
\hline \multicolumn{2}{|l|}{$R T$ dose } \\
\hline Median & $59.4 \mathrm{~Gy}$ \\
\hline Range & $12.6-70 \mathrm{~Gy}$ \\
\hline \multicolumn{2}{|l|}{$S U V \max$} \\
\hline Median & 15.6 \\
\hline Range & $7.3-51.6$ \\
\hline
\end{tabular}

$S C C$ squamous cell carcinoma, GEJ gastroesophageal junction,

$R T$ radiation therapy, $S U V$ standardized uptake value

\section{Concordance of semiautomatically delineated GTVs and manually delineated GTVs}

The semiautomatically delineated GTVs of the different methods were compared with the mean manually delineated $\mathrm{GTV}_{\mathrm{CT}}$ and $\mathrm{GTV}_{\mathrm{PET} / \mathrm{CT}}$ of the three observers by the Wilcoxon test.

Dice coefficients were computed for each patient for the semiautomatically delineated GTVs of every method with the manually delineated GTVs of every modality of the three observers separately. Mean Dice coefficients were then calculated for every observer and compared descrip- tively between the different methods of semiautomated delineation.

\section{Statistical analysis}

Statistical analysis was performed using the Statistical Package for Social Sciences (SPSS, version 26, SPSS Inc, Chicago, IL, USA). For descriptive analysis of patient characteristics and volumes, mean values and ranges were used. GTVs and tumor lengths were compared using the Wilcoxon test after testing for normality by the Kolgomorov-Smirnov test failed for the majority of parameters.

\section{Results}

A total of 45 [F18] FDG-PET/CT datasets of 45 patients were analyzed. Of these, 39 patients had SCC and 5 had histologically proven adenocarcinoma of the esophagus. Tumors were localized in the cervical (7 patients), upper thoracic (16 patients), middle thoracic (13 patients), and lower thoracic (9 patients) part of the esophagus. Of all patients, 16 had no evidence of lymph node involvement while 29 patients had positive lymph nodes on [F18] FDG-PET/CT. Detailed Patient characteristics are listed in Table 1.

\section{Interobserver variability}

Mean primary GTV volumes of all patients based on manual delineation on CT and PET/CT for each observer were 59.2 and $58.0 \mathrm{ml}$ for observer $\mathrm{A}, 65.4$ and $64.1 \mathrm{ml}$ for observer B, and 60.4 and $59.22 \mathrm{ml}$ for observer C. No significant difference between CT- and PET/CT-based delineation was found upon comparing the mean volumes of all three observers per patient on each modality $(60.4 \mathrm{ml}$ vs. $59.22 \mathrm{ml}, p=0.53$ ). Mean tumor length of all patients based on CT and PET/CT for each observer were 8.1 and $8.1 \mathrm{~cm}$ for observer A, 8.3 and $7.6 \mathrm{~cm}$ for observer $\mathrm{B}$, and 7.9 and $7.6 \mathrm{~cm}$ for observer C. Again, no significant difference between CT- and PET/CT-based delineation was found upon comparing the mean tumor lengths of all three observers per patient on each modality $(p=0.11)$, see Table 2 .

Mean DICE coefficients of all patients on CT and PET/CT were 0.79 and 0.77 for observer pair $\mathrm{AB}, 0.81$ and 0.78 for observer pair $\mathrm{AC}$, and 0.8 and 0.78 for observer pair BC. No significant difference was found between the mean Dice coefficients of all observer pairs per patient between CT- and PET/CT-based delineation (Table 2). 
Table 2 Comparison of manually delineated volumes of different modalities (GTV $\mathrm{GT}_{\mathrm{C}}$ vs. GTVPET/CT)

\begin{tabular}{|c|c|c|c|c|c|c|}
\hline \multirow{3}{*}{$\begin{array}{l}\text { Patient } \\
\text { Number }\end{array}$} & \multicolumn{2}{|c|}{ Three observers } & \multicolumn{2}{|c|}{ Three observers } & \multicolumn{2}{|c|}{ Three observer pairs } \\
\hline & Mean GTV & Mean GTV & Mean length & Mean length & Mean Dice & Mean Dice \\
\hline & $\mathrm{CT}(\mathrm{ccm})$ & $\mathrm{PET} / \mathrm{CT}(\mathrm{ccm})$ & $\mathrm{CT}(\mathrm{cm})$ & $\mathrm{PET} / \mathrm{CT}(\mathrm{cm})$ & $\mathrm{CT}$ & $\mathrm{PET} / \mathrm{CT}$ \\
\hline 1 & 488.15 & 488.57 & 14.83 & 15.27 & 0.82 & 0.86 \\
\hline 2 & 27.97 & 32.06 & 5.33 & 6.07 & 0.86 & 0.82 \\
\hline 3 & 182.09 & 175.51 & 16.83 & 16.17 & 0.89 & 0.87 \\
\hline 4 & 35.38 & 38.25 & 7.03 & 6.67 & 0.82 & 0.87 \\
\hline 5 & 65.04 & 64.16 & 8.63 & 9.20 & 0.85 & 0.83 \\
\hline 6 & 41.66 & 50.73 & 5.37 & 5.07 & 0.79 & 0.79 \\
\hline 7 & 21.41 & 18.35 & 4.03 & 3.63 & 0.81 & 0.81 \\
\hline 8 & 55.57 & 53.23 & 9.07 & 9.70 & 0.86 & 0.89 \\
\hline 9 & 25.03 & 21.96 & 7.80 & 5.13 & 0.86 & 0.81 \\
\hline 10 & 73.93 & 57.25 & 11.40 & 7.23 & 0.79 & 0.81 \\
\hline 11 & 13.98 & 16.07 & 4.50 & 4.20 & 0.78 & 0.79 \\
\hline 12 & 24.14 & 23.00 & 5.60 & 5.23 & 0.65 & 0.69 \\
\hline 13 & 26.57 & 27.20 & 7.30 & 6.67 & 0.84 & 0.81 \\
\hline 14 & 27.72 & 29.61 & 6.83 & 6.53 & 0.80 & 0.79 \\
\hline 15 & 7.50 & 13.82 & 2.83 & 4.23 & 0.76 & 0.55 \\
\hline 16 & 30.64 & 31.92 & 5.60 & 6.10 & 0.86 & 0.88 \\
\hline 17 & 37.98 & 37.93 & 7.33 & 6.57 & 0.90 & 0.82 \\
\hline 18 & 17.57 & 21.20 & 6.30 & 8.53 & 0.76 & 0.55 \\
\hline 19 & 24.57 & 28.27 & 6.90 & 7.47 & 0.78 & 0.77 \\
\hline 20 & 11.87 & 12.44 & 5.07 & 4.43 & 0.78 & 0.63 \\
\hline 21 & 39.53 & 39.84 & 7.20 & 6.83 & 0.82 & 0.78 \\
\hline 22 & 101.04 & 128.66 & 10.83 & 12.67 & 0.79 & 0.80 \\
\hline 23 & 7.50 & 6.41 & 4.67 & 3.00 & 0.82 & 0.86 \\
\hline 24 & 40.62 & 29.49 & 7.13 & 5.53 & 0.77 & 0.72 \\
\hline 25 & 13.99 & 16.62 & 4.57 & 5.13 & 0.79 & 0.74 \\
\hline 26 & 33.47 & 33.52 & 7.43 & 6.97 & 0.54 & 0.49 \\
\hline 27 & 11.66 & 9.87 & 5.10 & 4.43 & 0.48 & 0.61 \\
\hline 28 & 66.96 & 69.06 & 9.17 & 9.37 & 0.83 & 0.79 \\
\hline 29 & 160.32 & 165.15 & 10.57 & 9.17 & 0.84 & 0.89 \\
\hline 30 & 37.48 & 24.96 & 7.00 & 4.43 & 0.86 & 0.87 \\
\hline 31 & 23.34 & 18.28 & 7.33 & 5.50 & 0.81 & 0.68 \\
\hline 32 & 188.17 & 173.60 & 12.50 & 12.17 & 0.87 & 0.88 \\
\hline 33 & 137.50 & 132.84 & 12.33 & 10.87 & 0.85 & 0.90 \\
\hline 34 & 40.20 & 33.94 & 8.77 & 6.30 & 0.87 & 0.84 \\
\hline 35 & 23.65 & 24.74 & 3.80 & 4.13 & 0.87 & 0.82 \\
\hline 36 & 30.00 & 25.19 & 6.13 & 4.87 & 0.87 & 0.86 \\
\hline 37 & 27.56 & 26.71 & 6.00 & 7.07 & 0.83 & 0.74 \\
\hline 38 & 138.39 & 107.23 & 21.60 & 22.33 & 0.81 & 0.78 \\
\hline 39 & 65.53 & 90.83 & 7.17 & 6.47 & 0.76 & 0.72 \\
\hline 40 & 3.79 & 7.98 & 3.30 & 4.00 & 0.64 & 0.67 \\
\hline 41 & 55.96 & 54.61 & 7.53 & 7.80 & 0.88 & 0.86 \\
\hline 42 & 81.89 & 53.03 & 11.90 & 10.57 & 0.68 & 0.84 \\
\hline 43 & 57.95 & 54.82 & 9.80 & 11.70 & 0.85 & 0.81 \\
\hline 44 & 69.44 & 74.51 & 9.33 & 9.47 & 0.83 & 0.88 \\
\hline 45 & 22.46 & 21.53 & 6.87 & 6.20 & 0.85 & 0.82 \\
\hline Mean & 60.38 & 59.22 & 7.93 & 7.58 & 0.80 & 0.78 \\
\hline Min & 3.79 & 6.41 & 2.83 & 3.00 & 0.48 & 0.49 \\
\hline Max & 488.15 & 488.57 & 21.60 & 22.33 & 0.90 & 0.90 \\
\hline
\end{tabular}


Table 3 Volume comparison of manually and semiautomatically delineated GTVsp $=0.826$

\begin{tabular}{lll}
\hline & Mean GTV & Mean GTV \\
\hline GTET/CT \\
GTV SUV35 & $p<0.001$ & $p<0.001$ \\
GTV SUV40 & $p<0.001$ & $p<0.001$ \\
GTV Schaefer & $p<0.001$ & $p<0.001$ \\
GTV PERCIST-TLG & $p<0.001$ & $p<0.001$ \\
\hline
\end{tabular}

\section{Concordance of manually and semiautomatically delineated GTVs}

The mean SUVmax for the entire cohort was 17.3 (range 7.3-51.5). Mean values for SUV30, SUV35, SUV40, Schaefer's threshold, and PERCIST-TLG threshold were 5.3 (2.2.-15.5), 6.1 (2.6-18.1), 6.9 (2.9-20.6), 7.0 (3.4-19), and 3.0 (1.4-4.3), respectively. The corresponding mean GTV volumes for SUV30, SUV35, SUV40, Schaefer's threshold, and PERCIST TLG threshold were $69.1 \mathrm{ml}$ (2.6-22.5), $23.9 \mathrm{ml}(1.3-16.0), 18.8 \mathrm{ml}(0.7-14.1), 18.6 \mathrm{ml}$ (0.7-12.6), and $70.9 \mathrm{ml}(2.3-35.2)$, respectively.

Comparison of the semiautomatically delineated GTVs with the mean manually delineated $\mathrm{GTV}_{\mathrm{CT}}$ and $\mathrm{GTV}_{\mathrm{PET} / \mathrm{CT}}$ of all observers resulted in significant differences for all thresholds except for the comparison of PERCIST-TLG threshold with GTV $_{\text {СT }}$ or GTV PET/CT $_{\text {(Table 3). }}$

Mean Dice coefficients per observer were calculated for every semiautomatically delineated GTV with both man-

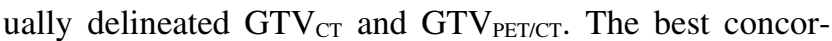
dance with the manually delineated GTV CT and GTV PET/CT was observed for PERCIST-TLG threshold (mean Dice $0.57-0.6$ with GTV CT and $0.61-0.65$ for PET/CT; Table 4).

The mean Dice coefficients per observer comparing the manually delineated $\mathrm{GTV}_{\mathrm{CT}}$ with the $\mathrm{GTV}_{\text {PET/CT }}$ were 0.77-0.8 (Table 5).

\section{Discussion}

Adequate delineation of the gross tumor volume of the primary tumor is a prerequisite for successful radiation treatment in general [21]. This is particularly important for
Table 5 Mean Dice similarity coefficient of manually delineated GTV CT vs. GTV PET/CT per observer

\begin{tabular}{llll}
\hline & Observer A & Observer B & Observer C \\
\hline Mean & 0.77 & 0.78 & 0.80 \\
Minimum & 0.46 & 0.46 & 0.42 \\
Maximum & 0.91 & 0.91 & 0.95 \\
\hline
\end{tabular}

the use of modern radiation techniques, with a high level of dose conformity (like IMRT or particle therapy) leading to a higher risk of suboptimal tumor coverage [21] in case of inadequate delineation. CT-based delineation incorporating information from other diagnostic modalities like endoscopy or endoscopic ultrasound is currently the standard approach for delineating the GTV of the primary tumor in esophageal cancer. However, the discriminative value of CT regarding tumor volume and surrounding normal esophageal or mediastinal tissue is often limited, as is the ability to relate spatial information from endoscopy or EUS to the planning CT. As most esophageal cancers show increased FDG uptake [21], especially in locally advanced cases which are typically treated with radiation therapy, it seemed reasonable to evaluate the incorporation of PET/CT into target volume delineation. Several groups have therefore investigated PET/CT-based delineation with regard to various endpoints with different methodologies (summarized in Table 6), thus (not surprisingly) reporting conflicting results $[6,15-20]$.

Direct correlation of imaging information with pathological specimens is the gold standard to test the validity of an imaging method and has also been studied in esophageal cancer [24-26]. However, this approach requires upfront surgery, which is not the preferred treatment option, especially for locally advanced esophageal cancer in the era of neoadjuvant chemo- or chemoradiation. Analysis of intra- and interobserver variability may serve as a surrogate based on the assumption that lower variability represents more accurate delineation [21].

Therefore, the first aim of our study was to evaluate if the addition of PET/CT to the standard approach based on CT combined with information from endoscopy reduces the interobserver variability of GTV definition regarding the primary tumor in a larger cohort than previously reported. We therefore compared the interobserver variability of three independent observers delineating the primary tumor in
Table 4 Mean Dice similarity coefficient comparing manually and semiautomatically delineated GTVs

\begin{tabular}{|c|c|c|c|c|c|c|c|c|}
\hline & \multicolumn{4}{|l|}{$\mathrm{GTV}_{\mathrm{CT}}$} & \multicolumn{4}{|c|}{ GTVPET/CT } \\
\hline & Obs. A & Obs. B & Obs. C & Mean & Obs. A & Obs. B & Obs. C & Mean \\
\hline SUV30 & 0.54 & 0.51 & 0.55 & 0.53 & 0.6 & 0.54 & 0.61 & 0.58 \\
\hline SUV35 & 0.52 & 0.49 & 0.53 & 0.51 & 0.57 & 0.51 & 0.59 & 0.56 \\
\hline SUV40 & 0.48 & 0.44 & 0.48 & 0.47 & 0.51 & 0.46 & 0.54 & 0.5 \\
\hline PERCIST-TLG & 0.59 & 0.57 & 0.6 & 0.59 & 0.64 & 0.61 & 0.65 & 0.63 \\
\hline Schaefer & 0.46 & 0.43 & 0.46 & 0.45 & 0.49 & 0.44 & 0.52 & 0.48 \\
\hline
\end{tabular}


Table 6 Overview on available literature

\begin{tabular}{|c|c|c|c|c|c|}
\hline Reference & Patients & Objective & SUV thresholds & $\begin{array}{l}\text { Reference } \\
\text { method }\end{array}$ & Conclusion \\
\hline $\begin{array}{l}\text { Schreurs } \\
\text { et al. [15] }\end{array}$ & $28 \mathrm{EC}$ & $\begin{array}{l}\text { Concordance indices GTV, } \\
\text { CTV, and PTV }\end{array}$ & None & $\mathrm{CT}+\mathrm{EUS}$ & $\begin{array}{l}\text { No statistically significant difference } \\
\text { in concordance indices } \\
\text { No impact on observer variation }\end{array}$ \\
\hline $\begin{array}{l}\text { Vali et al. } \\
{[16]}\end{array}$ & $22 \mathrm{EC}$ & $\begin{array}{l}\text { SUV threshold for GTV } \\
\text { delineation }\end{array}$ & $\begin{array}{l}\text { SUV2.0; SUV2.5; } \\
\text { SUV3.0; SUV3.5; } \\
\text { SUV40\%; SUV45\%; } \\
\text { SUV50\% }\end{array}$ & $\mathrm{CT}+\mathrm{EUS}$ & $\begin{array}{l}\text { SUV } 2.5 \text { yields the highest confor- } \\
\text { mality index and best approximates } \\
\text { the CT-based GTV at the epicenter }\end{array}$ \\
\hline $\begin{array}{l}\text { Dong et al. } \\
{[17]}\end{array}$ & $\begin{array}{l}50 \text { SCEC; } \\
50 \text { NSCLC }\end{array}$ & $\begin{array}{l}\text { Influence of uptake hetero- } \\
\text { geneity on tumor delin- } \\
\text { eation }\end{array}$ & SUV40\%; SUV2.5 & CT & $\begin{array}{l}\text { Larger GTV delineation difference } \\
\text { in tumors with high FDG uptake } \\
\text { heterogeneity }\end{array}$ \\
\hline $\begin{array}{l}\text { Thomas } \\
\text { et al. [18] }\end{array}$ & $20 \mathrm{EC}$ & $\begin{array}{l}\text { Tumor volume, tumor } \\
\text { length, and volume over- } \\
\text { lap }\end{array}$ & $\begin{array}{l}\text { SUV2.0; SUV2.5; } \\
\text { SUV3.0; SUV20\%; } \\
\text { SUV35\%; SUV40\%; } \\
\text { SUV45\% }\end{array}$ & $\mathrm{CT}+$ clips & $\begin{array}{l}\text { CT + clips as "gold standard," no } \\
\text { close agreement with CT alone or } \\
\text { PET/CT }\end{array}$ \\
\hline $\begin{array}{l}\text { Nowee } \\
\text { et al. [24] }\end{array}$ & $6 \mathrm{EC}$ & $\begin{array}{l}\text { Interobserver variation, CI, } \\
\text { most cranial/caudal slice }\end{array}$ & None & $\begin{array}{l}\text { CT, clinical } \\
\text { data, EUS }\end{array}$ & Limited impact on observer variation \\
\hline $\begin{array}{l}\text { Jimenez- } \\
\text { Jimenez } \\
\text { et al. [19] }\end{array}$ & $29 \mathrm{EC}$ & $\begin{array}{l}\text { GTVtumor and GTVnode } \\
\text { comparison of volume and } \\
\text { tumor length }\end{array}$ & None & $\mathrm{CT}$ & $\begin{array}{l}\text { No significant difference in volume } \\
\text { of GTVtumor but in GTVnode }\end{array}$ \\
\hline $\begin{array}{l}\text { Toya et al. } \\
{[20]}\end{array}$ & $10 \mathrm{CEC}$ & Interobserver variation & None & $\begin{array}{l}\text { ceCT, barium } \\
\text { esophagogram, } \\
\text { EUS }\end{array}$ & $\begin{array}{l}\text { PET/CT may increase consistency } \\
\text { in GTV delineation in patients with } \\
\text { CEC }\end{array}$ \\
\hline
\end{tabular}

$E C$ esophageal cancer, $S C E C$ squamous cell esophageal cancer, $C E C$ cervical esophageal cancer, $E U S$ endoscopic ultrasound, $V R$ volume ratio, $C I$ conformity index, $D I$ degree of inclusion

45 cases either with CT or PET/CT with regard to volume and length of corresponding GTVs. Surprisingly, we were not able to detect significant differences between the delineated volumes or the tumor lengths between the two methods. Moreover, concordance between the observers measured by DICE coefficients was not significantly different between the methods, with the absolute values even favoring the CT-based approach (mean Dice coefficient 0.8 vs. 0.78 for CT vs. PET/CT based delineation). These results are in line with the findings of several smaller studies using similar approaches. For example, Scheurs et al. [15] evaluated 28 patients comparing CT- and PET/CT-based delineation by three observers using a concordance index and reported no significant differences. Nowee et al. [6] evaluated 6 cases with 20 observers in a nationwide study in the Netherlands using a conformity index and similarly found no significant reduction in interobserver variability between the two modalities. In contrast, Toya et al. [20] described a significantly reduced interobserver variability for PET/CT compared to CT measured by conformality index in their cohort of 10 patients with cervical esophageal cancer delineated by five observers, with significantly smaller GTVs based on PET/CT imaging. Similarly, Vesprini et al. [27] reported a small but significant reduction in intraobserver variability for the PET/CT-based approach in their study of 10 patients with gastroesophageal cancer. In summary, a benefit of using PET/CT for delineation of the primary tumor volume remains questionable based on the available literature, especially given the negative results of the larger studies including ours. These recent findings confirm the results of a systematic review published by Mujis et al. [21] in 2010, which similarly concluded that the (at that time more limited) available data did not provide sufficient evidence that the integration of PET/CT will necessarily improve the accuracy of GTV delineation in patients with esophageal cancer.

However, these findings should not be misinterpreted regarding PET/CT as a useless tool per se in radiation therapy planning for esophageal cancer. Several groups have described the superiority of PET/CT compared to conventional CT in the detection and delineation of affected lymph nodes, which may lead to substantial changes in nodal GTVs and CTVs [13, 19, 28-30], although inconsistent data from imaging studies on the improvement of sensitivity and specificity of PET/CT compared to other staging modalities exist $[21,31]$. Recently, a retrospective analysis of 145 patients treated with neoadjuvant or definitive chemoradiation for esophageal cancer compared patients with or without PET/CT staging with regard to outcome [31]. They found a significantly improved locoregional recurrence-free survival in patients staged by PET/CT with a trend even to improved survival probably based on more accurate target delineation and consequently improved treatment efficacy [31]. Moreover, PET/CT clearly improves the detection of distant metastases compared to conventional CT $[32,33]$ 
and therefore seems crucial for adequate patient selection for localized therapies like radiation [31, 33].

Some reasons for the lack of improvement by the addition of PET/CT for delineation of the primary GTV might be simply technical issues. Delineated volumes based on PET/CT may vary extensively based on the contouring method using the metabolic information. Two major types of contouring methods exist, either using visual interpretation (with or without source-to-background correction) or different fixed SUV thresholds. Visual interpretation, which was used in most of the cited studies, is highly observer dependent as image representation can be controlled by changing window widths or window levels, resulting in different visible tumor volumes [21]. On the other hand, SUV as a semiquantitative parameter can be affected by many parameters such as patient preparation, scan acquisition, image reconstruction, and data analysis, which may result in considerable differences in SUV outcome even though most of these effects are small [21]. Nevertheless, semiautomated delineation methods based on fixed thresholds may be helpful in the harmonization of GTV volume definition.

The second aim of our study was therefore to evaluate different SUV-dependent methods with regard to their concordance with manually delineated GTV volumes. Different SUV-based thresholds have been analyzed in the past, either based on fixed absolute values like SUV2.5 [16], based on relative SUV values like SUV30 which represents 30\% of the SUVmax of the individual patient $[16,18]$, or even more complex thresholds including corrections for background or metabolic activity [10]. As thresholds based on absolute values might be additionally influenced by individual patient parameters such as body weight and blood glucose levels, we decided to evaluate relative SUV values (SUV30, 35, and 40). Based on our initial experience, we included the background- and metabolically corrected PERCIST-TLG [10] and Schaefer's algorithm [22]. Our previous work had described especially the PERCIST TLG algorithm as promising in an analysis including 20 patients with solid tumors including 5 suffering from esophageal cancer [10].

As assumed, we observed considerable differences in the GTV volumes derived from semiautomated delineation with different thresholds, which varied distinctly not only between each other but also compared to the manually delineated GTVs based on CT and PET/CT using visual interpretation. Indeed, all thresholds used resulted in significantly different volumes compared to the mean manually delineated GTVs of the three observers both for CT and PET/CT, except the PERCIST-TLG algorithm.

Regarding the calculated Dice coefficients for the different semiautomatically delineated volumes with the manually delineated volumes of the three observers, we found the highest mean Dice coefficients for the PERCIST-TLG algorithm with manually derived CT (mean Dice coefficient 0.59) as well as PET/CT GTVs (mean Dice coefficient 0.63). However, even the best semiautomated volume (PERCIST-TLG) had a lower concordance with both manually derived GTVs (CT vs. PET/CT) than the GTV PET/CT $_{\text {based }}$ on visual interpretation with the $\mathrm{GTV}_{\mathrm{CT}}$ for every observer (mean Dice coefficient 0.79), indicating a still suboptimal result.

One major drawback for the use of semiautomatically delineated volumes is the common practice of radiation oncologists to always include the whole circumference of the esophagus into the GTV even if visible primary tumor is present only at one side of the esophageal wall. In contrast, all software-based algorithms will contour volumes strictly restricted to detected tumor, which sometimes result in GTVs not covering the whole esophageal circumference (as illustrated in Fig. 4). This issue should be addressed in the design of further studies, either by adding a step to automated contouring to always include the whole esophageal structure in the corresponding CT slice if tumor is automatically detected, or in advising the human observers to strictly contour only the visible tumor regardless of the adjacent esophageal structure.

Our study has some limitations: It is of retrospective nature and therefore may not serve as a confirmative but rather as a hypothesis-generating study regarding at least the design of future studies. Due to the high number of included patients, we decided to limit the number of observers to three, which seems to be the possible minimum (although studies with larger numbers of observers provided similar results). As we focused on primary gross tumor volume, no insights into the possible value of PET/CT for lymph node delineation, clinical target volume definition, or detection of distant metastases can be given. Finally, the common practice of radiation oncologists to include the whole circumference of the esophagus into the GTV even if visible tumor (on imaging) is present only at parts of the circumference makes direct comparisons to semiautomatically detected volumes difficult.

\section{Conclusion}

In summary, we were not able to show that the integration of PET/CT for GTV delineation of the primary tumor in esophageal cancer patients resulted in reduced interobserver variability in a large cohort of patients. Moreover, the evaluated semiautomatically delineated GTVs based on fixed SUV thresholds did not correlate well with the manually derived GTV volumes based either on CT or visually interpreted PET/CT. Nevertheless, we found that the most promising algorithms for further evaluation of semiautomatically delineated volumes probably seem to be back- 
ground- and metabolically corrected algorithms like PERCIST-TLG, which showed the highest concordance with the manually derived GTVs of all evaluated methods. However, our findings should not be misinterpreted regarding the general value of PET/CT for staging and treatment planning in esophageal cancer patients based on the possible advantages for detection of lymph nodes, including definition of clinical target volumes or the detection of distant metastases. Further studies regarding primary GTV definition should account for general differences between human and semiautomated contouring with regard to inclusion of the whole circumference of tumor-bearing slices of the esophageal structure.

Author Contribution FW participated in patient treatment, data acquisition, statistical analysis, and in drafting the manuscript. CJ and HI performed data acquisition and participated in statistical analysis. FW, BZ, and CA performed $\mathrm{GTV}_{\mathrm{CT}}$ and $\mathrm{GTV}_{\mathrm{PET} / \mathrm{CT}}$ delineation. MN and $\mathrm{CB}$ critically reviewed the manuscript. FR participated in data acquisition, statistical analysis, treatment of the patients, drafting the manuscript, and critically reviewed the data and the manuscript.

Funding Open Access funding enabled and organized by Projekt DEAL.

\section{Compliance with ethical guidelines}

Conflict of interest All authors declare that they have no competing interests.

Ethical standards The study was approved by the Ethics committee of the University of Munich (LMU), reference number 634-16.

Open Access This article is licensed under a Creative Commons Attribution 4.0 International License, which permits use, sharing, adaptation, distribution and reproduction in any medium or format, as long as you give appropriate credit to the original author(s) and the source, provide a link to the Creative Commons licence, and indicate if changes were made. The images or other third party material in this article are included in the article's Creative Commons licence, unless indicated otherwise in a credit line to the material. If material is not included in the article's Creative Commons licence and your intended use is not permitted by statutory regulation or exceeds the permitted use, you will need to obtain permission directly from the copyright holder. To view a copy of this licence, visit http://creativecommons.org/licenses/by/4. $0 /$.

\section{References}

1. Roeder F et al (2014) Intensity modulated radiotherapy (IMRT) with concurrent chemotherapy as definitive treatment of locally advanced esophageal cancer. Radiat Oncol 9:191

2. Haefner MF et al (2018) Neoadjuvant versus definitive chemoradiotherapy for locally advanced esophageal cancer: outcomes and patterns of failure. Strahlenther Onkol 194(2):116-124

3. Herskovic A et al (1992) Combined chemotherapy and radiotherapy compared with radiotherapy alone in patients with cancer of the esophagus. N Engl J Med 326(24):1593-1598

4. van Hagen P et al (2012) Preoperative chemoradiotherapy for esophageal or junctional cancer. N Engl J Med 366(22):2074-2084
5. Wu AJ et al (2015) Expert consensus contouring guidelines for intensity modulated radiation therapy in esophageal and gastroesophageal junction cancer. Int J Radiat Oncol Biol Phys 92(4):911-920

6. Nowee ME et al (2019) Gross tumour delineation on computed tomography and positron emission tomography-computed tomography in oesophageal cancer: a nationwide study. Clin Transl Radiat Oncol 14:33-39

7. Walter F et al (2018) Clinical outcome of elderly patients ( $\geq$ 70 years) with esophageal cancer undergoing definitive or neoadjuvant radio(chemo)therapy: a retrospective single center analysis. Radiat Oncol 13(1):93

8. Hegemann NS et al (2018) Neoadjuvant chemoradiation for esophageal cancer: surgery improves locoregional control while response based on FDG-PET/CT predicts survival. Strahlenther Onkol 194(5):435-443

9. Grosu AL et al (2005) Positron emission tomography for radiation treatment planning. Strahlenther Onkol 181(8):483-499

10. Niyazi $\mathrm{M}$ et al (2013) Automated biological target volume delineation for radiotherapy treatment planning using FDG-PET/CT. Radiat Oncol 8:180

11. Eze C et al (2019) Initial report on feasibility of PET/CT-based image-guided moderate hypofractionated thoracic irradiation in nodepositive non-small cell lung cancer patients with poor prognostic factors and strongly diminished lung function: a retrospective analysis. Radiat Oncol 14(1):163

12. Schmidt-Hegemann NS et al (2018) Outcome after PSMA PET/CT based radiotherapy in patients with biochemical persistence or recurrence after radical prostatectomy. Radiat Oncol 13(1):37

13. Yuan $\mathrm{S}$ et al (2006) Additional value of PET/CT over PET in assessment of locoregional lymph nodes in thoracic esophageal squamous cell cancer. J Nucl Med 47(8):1255-1259

14. Machiels M et al (2016) Distribution of lymph node metastases on FDG-PET/CT in inoperable or unresectable oesophageal cancer patients and the impact on target volume definition in radiation therapy. J Med Imaging Radiat Oncol 60(4):520-527

15. Schreurs LM et al (2010) Impact of 18-fluorodeoxyglucose positron emission tomography on computed tomography defined target volumes in radiation treatment planning of esophageal cancer: reduction in geographic misses with equal inter-observer variability: PET/CT improves esophageal target definition. Dis Esophagus 23(6):493-501

16. Vali FS et al (2010) Comparison of standardized uptake valuebased positron emission tomography and computed tomography target volumes in esophageal cancer patients undergoing radiotherapy. Int J Radiat Oncol Biol Phys 78(4):1057-1063

17. Dong $X$ et al (2015) Intra-tumour 18F-FDG uptake heterogeneity decreases the reliability on target volume definition with positron emission tomography/computed tomography imaging. J Med Imaging Radiat Oncol 59(3):338-345

18. Thomas L et al (2015) Tumour delineation in oesophageal cancer-a prospective study of delineation in PET and CT with and without endoscopically placed clip markers. Radiother Oncol 116(2):269-275

19. Jimenez-Jimenez E et al (2018) Radiotherapy volume delineation using 18F-FDG-PET/CT modifies gross node volume in patients with oesophageal cancer. Clin Transl Oncol 20(11):1460-1466

20. Toya $\mathrm{R}$ et al (2019) Impact of hybrid FDG-PET/CT on gross tumor volume definition of cervical esophageal cancer: reducing interobserver variation. J Radiat Res 60(3):348-352

21. Muijs CT et al (2010) A systematic review on the role of FDG$\mathrm{PET} / \mathrm{CT}$ in tumour delineation and radiotherapy planning in patients with esophageal cancer. Radiother Oncol 97(2):165-171

22. Schaefer A et al (2012) Multi-centre calibration of an adaptive thresholding method for PET-based delineation of tumour vol- 
umes in radiotherapy planning of lung cancer. Nuklearmedizin 51(3): 101-110

23. Nowee ME et al (2019) Gross tumour delineation on computed tomography and positron emission tomography-computed tomography in oesophageal cancer: A nationwide study. Clin Transl Radiat Oncol 14:33-39

24. Zhong X et al (2009) Using 18F-fluorodeoxyglucose positron emission tomography to estimate the length of gross tumor in patients with squamous cell carcinoma of the esophagus. Int J Radiat Oncol Biol Phys 73(1):136-141

25. Han D et al (2010) Comparison of (18)F-fluorothymidine and (18)F-fluorodeoxyglucose PET/CT in delineating gross tumor volume by optimal threshold in patients with squamous cell carcinoma of thoracic esophagus. Int J Radiat Oncol Biol Phys 76(4):1235-1241

26. Yu W et al (2009) GTV spatial conformity between different delineation methods by 18FDG PET/CT and pathology in esophageal cancer. Radiother Oncol 93(3):441-446

27. Vesprini D, Ung Y, Dinniwell R et al (2008) Improving observer variability in target delineation for gastro-oesophageal cancer-the role of 18F-fluoro-2-deoxy-D-glucose positron emission tomography/computed tomography. Clin Oncol 20:631-638
28. Sihvo EI, Rasanen JV, Knuuti MJ et al (2004) Adenocarcinoma of the esophagus and the esophagogastric junction: positron emissio tomography improves staging and prediction of survival in distant but not in locoregional disease. J Gastrointest Surg 8:988-996

29. Scheurs LM, Pultrum BB, Koopmans KP et al (2008) Better assessment of nodal metastases by PET/CT fusion compared to side-byside PET/CT in oesophageal cancer. Anticancer Res 28:1867-1873

30. Kato H, Kimura H, Nakajima $M$ et al (2008) The additional value of integrated PET/CT over PET in initial lymph node staging of esophageal cancer. Oncol Rep 20:857-861

31. Metzger JG, Wollschläger D, Miederer M, Vaupel P, Moehler M, Schmidberger H, Mayer A (2017) Inclusion of PET-CT into planning of primary or neoadjuvant chemoradiotherapy of esophageal cancer improves prognosis. Strahlenther Onkol 193:791-799

32. van Westreenen HL, Westerterp M, Bossyut PM et al (2004) Systematic review of the staging performance of 18-F-fluorodeoxyglucose positron emission tomographys in esophageal cancer. J Clin Oncol 22:3805-3812

33. Chatterton BE, Shon IH, Baldey A et al (2009) Positron emission tomography changes management and prognostic stratification in patients with oesophageal cancer: results of a multicenter prospective study. Eur J Nucl Med Mol Imaging 36:354-361 